\title{
Variáveis que Podem Interferir no Seguir Regras de Participantes Flexíveis e Inflexíveis
}

\author{
Variables which May Interfere in Following Rules \\ by Flexible and Inflexible Participants
}

\author{
Carla Cristina Paiva Paracampo*, Lívia Mello Souza \& Luiz Carlos de Albuquerque \\ Universidade Federal do Pará, Belém, Pará, Brasil
}

\begin{abstract}
Resumo
Com o objetivo de avaliar o efeito de histórias pré-experimentais sobre o seguir regras, 16 estudantes universitários foram expostos a um procedimento informatizado de escolha segundo o modelo. A tarefa era apontar cada um dos três estímulos de comparação em sequência. Oito participantes, quatro flexíveis e quatro inflexíveis, foram submetidos a cada uma das duas condições de quatro fases. $\mathrm{Na}$ Fase 1, a sequência correta era estabelecida por contingências. Reforço para a emissão da sequência correta foi mantido durante as três fases - na Condição 1, o programa de reforço contínuo (CRF) e na Condição 2, a relação fixa de quatro (FR4). Em ambas as condições, a Fase 2 começou com a apresentação de uma regra discrepante, a Fase 3 começou com uma regra correspondente, e Fase 4 começou com uma regra discrepante. Seguindo a regra discrepante não produziu o reforço programado, mas seguindo a regra produzida correspondente reforço. Na Condição 1 (CRF) , os 8 participantes deixaram de seguir a regra discrepante, independentemente de serem flexíveis ou inflexíveis. $\mathrm{Na}$ Condição 2 (FR4), os inflexíveis seguiram e os flexíveis deixaram de seguir a regra discrepante. Sugere-se que os efeitos de histórias pré-experimentais são modulados por variáveis atuais.

Palavras-chave: Regras, contingências, diferenças individuais, participantes flexíveis e inflexíveis, histórias pré-experimentais, histórias experimentais.
\end{abstract}

\begin{abstract}
Aiming to evaluate the effect of pre-experimental histories on rule-following, 16 college students were submitted to a computerized matching-to-sample procedure. The task was to point to each of three comparison stimuli in sequence. Eight participants, 4 flexible and 4 inflexible, were allocated to each of two four-phase conditions. In Phase 1, the correct sequence was established by contingencies. Reinforcement to the emission of the correct sequence was maintained during the following three phases - in Condition 1, the continuous reinforcement schedule (CRF), and in Condition 2, the fixed-ratio 4 (FR4). In both conditions, Phase 2 started with the presentation of a discrepant rule, Phase 3 started with a corresponding rule, and Phase 4 started with a discrepant rule. Following the discrepant rule did not produce the programmed reinforcement, but following the corresponding rule produced reinforcement. In Condition 1 (CRF), the 8 participants, flexible and inflexible, stopped following the discrepant rule. In Condition 2 (FR 4), the inflexible participants kept following the discrepant rule and the flexible ones stopped following it. It is suggested that the effects of pre-experimental histories are modulated by present variables.

Keywords: Rules, contingencies, individual differences, flexible and inflexible participants, pre-experimental histories, experimental histories.
\end{abstract}

Regras são estímulos antecedentes verbais que podem descrever contingências; estabelecer comportamentos novos independentemente de suas consequências imediatas; e, alterar as funções de estímulos (Albuquerque, 2001, 2005; Albuquerque \& Paracampo 2010). Quando o comportamento é controlado por regras, são as regras que colocam o comportamento sob o controle de estímulos, determinam a sua topografia, a sua probabilidade de ocorrer no futuro e alteram as funções dos estímulos. Já

\footnotetext{
" Endereço para correspondência: Rua Oliveira Belo, 238, Apto. 1702, Umarizal, Belém, PA, Brasil 66050-380. E-mail: cparacampo@gmail.com
}

quando o comportamento é controlado por contingências, são as consequências imediatas do comportamento que exercem essas funções (Albuquerque, 2001, 2005; Albuquerque \& Paracampo, 2010; Albuquerque, Souza, Matos, \& Paracampo, 2003).

Os estudos que têm investigado o controle por regras (para uma revisão, ver Baron \& Galizio, 1983; Paracampo \& Albuquerque, 2005), de modo geral, têm contribuído para esclarecer como o ambiente verbal, seja público ou privado, funciona e de que maneira ele exerce controle sobre o comportamento humano. Por essa visão, além de poder exercer as funções de consequências imediatas do comportamento, o ambiente verbal também pode exercer as funções de regras (Albuquerque, 2005). 
Um problema, no entanto, que ainda não está claro nessa área de pesquisa, é a variabilidade nos resultados (relativos ao seguimento e ao não-seguimento de regras), frequentemente encontrada, dentro de uma mesma condição experimental, em alguns dos estudos (por exemplo, Albuquerque et al., 2003; Hayes, Brownstein, Zettle, Rosenfarb, \& Korn, 1986; LeFrancois, Chase, \& Joyce, 1988; Newman, Buffington, \& Hemmes, 1995; Oliveira \& Albuquerque, 2007; Paracampo \& Albuquerque, 2004; Shimoff, Catania, \& Matthews, 1981).

Em geral, diferenças entre os desempenhos de participantes humanos dentro de uma mesma condição experimental, têm sido atribuídas, principalmente, a efeitos de histórias (Weiner, 1983). Uma explicação, então, para tais diferenças, encontradas em alguns dos estudos da área, seria que pessoas que tiveram o comportamento de não seguir regras punido socialmente em uma variedade de situações, ao longo de suas histórias pré-experimentais, tenderiam a seguir regras. Já pessoas que tiveram o comportamento de não seguir regras frequentemente reforçado socialmente, tenderiam a não seguir regras, inclusive em situações experimentais (Wulfert, Greenway, Farkas, Hayes, \& Douguer, 1994).

Wulfert e colaboradores (1994) sugeriram que os efeitos de tais histórias pré-experimentais poderiam ser inferidos de respostas dos participantes ao questionário desenvolvido por Rehfisch (1958), denominado de Escala de Rigidez. Ainda de acordo com Wulfert e colaboradores, esta escala permitiria identificar indivíduos com tendência a seguir regras devido a uma história pré-experimental de punição do não seguimento de regras e identificar indivíduos com tendência a não seguir regras devido a uma história pré-experimental de reforço do não seguimento de regras, principalmente, porque a escala tem validade. Além disso, ela conteria itens, tais como: "Eu sempre sigo a regra: negócios antes do prazer" (Item 9 do questionário). E porque, diante de uma afirmação como a do Item 9, alguns participantes tenderiam a relatar que é verdadeira (os participantes que tendem a concordar com afirmações como esta são precisamente aqueles que, ao final da aplicação do questionário, são classificados de inflexíveis) e outros tenderiam a relatar que é falsa (os participantes que tendem a discordar de afirmações como esta são precisamente aqueles, que ao final da aplicação do questionário, são classificados de flexíveis).

Considerando isto, Wulfert e colaboradores (1994) procuraram investigar se os autorrelatos indicativos de flexibilidade (participantes flexíveis) e os autorrelatos indicativos de inflexibilidade (participantes inflexíveis), identificados a partir da aplicação da Escala de Rigidez desenvolvida por Rehfisch (1958), podem ser utilizados para se prever a manutenção do seguir regras discrepantes das contingências programadas em situações experimentais. Observaram que participantes (estudantes universitários) com repertórios classificados de inflexíveis foram mais prováveis de seguirem a regra discrepante das contingências do esquema múltiplo a que foram expostos do que os participantes classificados de flexíveis.

Pinto, Paracampo e Albuquerque (2006), procuraram comparar os efeitos, sobre o seguimento de regras discrepantes das contingências, de histórias pré-experimentais (proposição de Wulfert e colaboradores, 1994, que sugere que participantes inflexíveis tendem a seguir regras discrepantes das contingências) e de histórias experimentais (proposição de Albuquerque \& Silva, 2006, que sugere que o seguimento de regras discrepantes das contingências tende a deixar de ocorrer, quando, antes da apresentação da regra discrepante, é construída uma história experimental em que o comportamento alternativo ao especificado pela regra mostra-se sob o controle das contingências, isto é, muda acompanhando a mudança nas contingências). Para tanto, expuseram estudantes universitários, previamente classificados como flexíveis e como inflexíveis, a um procedimento de escolha de acordo com o modelo, similar ao usado por Albuquerque e colaboradores (2003), e encontraram resultados similares aos resultados obtidos no estudo de Wulfert e colaboradores (1994). Ou seja, os participantes inflexíveis tenderam a seguir a regra discrepante e o os participantes flexíveis tenderam a deixar de seguir tal regra.

Pinto, Paracampo e Albuquerque (2008) notaram, no entanto, que os estudos de Pinto e colaboradores (2006) e Wulfert e colaboradores (1994) não deixam claro se o seguir a regra discrepante foi mantido, porque os participantes tinham uma história pré-experimental de reforço para o seguir e de punição para o não seguir regras (isto é, porque eles eram inflexíveis) ou porque o seguir regras não produzia perda de reforçadores. Isto não fica claro porque os estudos de Pinto e colaboradores (2006) e Wulfert e colaboradores (1994) não investigaram essa possibilidade.

Considerando isto, Pinto e colaboradores (2008) procuraram verificar se participantes previamente classificados de inflexíveis tem maior probabilidade de seguir regras discrepantes das contingências do que participantes previamente classificados de flexíveis, quando o seguir regras discrepantes produz perda de reforçadores. Os participantes (estudantes universitários) foram expostos a um procedimento de escolha de acordo com o modelo, similar ao usado por Albuquerque colaboradores (2003). Dez (5 flexíveis e 5 inflexíveis) dos 11 participantes deixaram de seguir regra discrepante das contingências programadas. De acordo com os autores, os resultados sugerem que o seguimento de regra discrepante tende a deixar de ocorrer quando produz perda de reforçadores, independentemente de se o participante é classificado de flexível ou de inflexível.

Os resultados dos estudos de Pinto e colaboradores (2006) e Wulfert colaboradores (1994) indicam que as histórias pré-experimentais dos participantes (isto é, suas classificações em inflexível e flexível), inferidas de suas respostas ao questionário desenvolvido por Rehfisch (1958), podem contribuir, em parte, para prever a ocorrência de diferenças entre desempenhos observadas dentro de uma mesma condição experimental. Contudo, os resultados 
do estudo de Pinto colaboradores (2008) indicam que os eventuais efeitos de histórias pré-experimentais dos participantes dependem, em parte, das condições atuais às quais o ouvinte é exposto.

De acordo com alguns autores (Albuquerque et al., 2003; Oliveira \& Albuquerque, 2007), a variabilidade nos resultados, encontrada em alguns estudos, não ocorre em toda e qualquer condição, uma vez que ela tende a ocorrer, com maior frequência, em condições que favorecem, de forma mais equilibrada, tanto a manutenção quanto o abandono do seguimento de regra. Para estes autores, os eventuais efeitos das histórias pré-experimentais sobre o comportamento dependem da situação atual a qual este comportamento está exposto.

Uma maneira de testar essa proposição seria expor participantes previamente classificados de inflexíveis e de flexíveis, de acordo com as suas respostas ao questionário desenvolvido por Rehfisch (1958), a uma condição em que frequentemente se nota uma tendência a não ocorrer variabilidade (condição sem variabilidade) e a outra condição em que frequentemente se nota uma tendência a ocorrer variabilidade nos resultados (condição com variabilidade).

Assim, se os resultados da condição sem variabilidade fossem replicados, independentemente da classificação dos participantes, e se os resultados da condição com variabilidade também fossem replicados, mas fosse constatado que os participantes que seguiram a regra haviam sido classificados de inflexíveis e os participantes que deixaram de seguir a regra haviam sido classificados de flexíveis, poder-se-ia especificar as condições sob as quais uma história pré-experimental teria maior ou menor probabilidade de exercer os seus eventuais efeitos.

Por outro lado, se tanto na condição sem variabilidade quanto na condição com variabilidade, fosse constatado que os participantes que haviam sido classificados de inflexíveis seguiram a regra e os participantes que haviam sido classificados de flexíveis deixaram de seguir a regra, poder-se-ia supor que os efeitos de histórias pré-experimentais, inferidos das respostas dos participantes ao questionário desenvolvido por Rehfisch (1958), podem ocorrer independentemente das condições experimentais investigadas. $\mathrm{O}$ presente estudo procurou testar essas possibilidades.

Para tanto, no presente estudo, como condição com variabilidade, foi usado o procedimento do Experimento 2 do estudo de Albuquerque e colaboradores (2003) e, como condição sem variabilidade, foi usado o procedimento da Condição 2 do Experimento 2 do estudo de Albuquerque, Reis e Paracampo (2006) ${ }^{1}$. Nestes dois estudos, os participantes foram expostos a um procedimento de escolha de acordo com o modelo. O comportamento alternativo ao especificado pela regra foi estabelecido por reforço diferencial (isto é, por contingências) em CRF (esquema de reforço contínuo) e depois foi mantido em FR 4 (razão

${ }^{1}$ Para facilitar a descrição, a partir desse momento estes dois estudos serão denominados de Albuquerque et al. (2006) [ou estudo de 2006] e Albuquerque et al. (2003) [ou estudo de 2003], respectivamente. fixa 4), no estudo de 2003, e em CRF, no estudo de 2006. Em seguida, nos dois estudos, foi apresentada uma regra discrepante das contingências programadas. Esta regra relatava que se o participante emitisse o comportamento por ela especificado, ele ganharia pontos trocáveis por dinheiro (reforço programado). Mas o comportamento que produzia o reforço programado era o previamente estabelecido por contingências, e não o seguir a regra discrepante. Portanto, estes dois estudos diferiam quanto ao esquema de reforço usado na fase de estabelecimento do comportamento alternativo ao especificado pela regra discrepante. No estudo de 2003 (manutenção do comportamento alternativo em FR 4), houve variabilidade, uma vez que quatro participantes seguiram e dois deixaram de seguir a regra discrepante. No estudo de 2006 (manutenção do comportamento alternativo em CRF), não houve variabilidade, uma vez que todos os quatro participantes deixaram de seguir a regra discrepante.

O presente estudo diferiu de cada um dos dois estudos anteriores (Albuquerque et al. 2006; Albuquerque et al., 2003), primeiro, porque ele apresenta as características desses dois estudos (e não apenas de um), como a utilização dos esquemas CRF e FR; segundo, porque os participantes foram previamente classificados de inflexíveis e de flexíveis; $\mathrm{e}$, terceiro, porque foi usada uma versão informatizada do procedimento de escolha de acordo com o modelo usado por Albuquerque e colaboradores (2003).

Considerando isto, o presente estudo teve como objetivo verificar, experimentalmente, se os efeitos de histórias pré-experimentais, inferidas das respostas dos participantes ao questionário sobre inflexibilidade, dependem da condição atual à qual o participante é exposto.

\section{Método}

\section{Participantes}

$\mathrm{Na}$ primeira etapa deste estudo (exposição dos participantes ao questionário sobre inflexibilidade) foram convidados, por meio de um convite oral, 175 estudantes universitários, sem história experimental prévia neste tipo de pesquisa, de diversos cursos (exceto o de Psicologia), e matriculados em diferentes semestres. Desses 175, 16 participantes ( 8 flexíveis e 8 inflexíveis) foram convidados a participar da segunda etapa da pesquisa (exposição dos participantes às regras e às contingências programadas). Antes do início da primeira etapa do estudo, os 175 participantes desta etapa leram e assinaram o Termo de Consentimento Livre e Esclarecido referente a primeira etapa do estudo. Antes do início da segunda etapa do estudo, os 16 participantes desta etapa leram e assinaram o Termo de Consentimento Livre e Esclarecido referente a segunda etapa do estudo.

\section{Material e Equipamentos}

Na primeira etapa do estudo foi utilizado o questionário, construído por Rehfisch (1958), denominado de Escala de Rigidez, o qual é baseado no Minnesota Multiphasic 
Paracampo, C. C. P., Souza, L. M. \& Albuquerque, L. C. (2014). Variáveis que Podem Interferir no Seguir Regras de Participantes Flexíveis e Inflexíveis.

Personality Inventory e no California Personality Inventory. Este questionário é constituído de 39 itens do tipo verdadeiro / falso.

Na segunda etapa do estudo foi utilizado um computador com um programa (software) desenvolvido em ambiente Windows especialmente para uso da pesquisa, de acordo com seus objetivos. O programa, uma versão informatizada do procedimento usado por Albuquerque e colaboradores (2003), foi produzido para apresentar as instruções, as contingências programadas e registrar as respostas dos participantes.

Durante o experimento, os estímulos apareciam na tela do computador. Acima e ao centro da tela, havia um contador que registrava os pontos. Abaixo do contador, havia uma caixa de texto onde eram apresentadas as instruções, bem como a consequência verbal ("Você ganhou um ponto") que seguia a sequência de respostas (correta) que cumpria a contingência programada. As instruções foram gravadas em um pen drive e eram apresentadas aos participantes por intermédio das caixas de som. Abaixo da caixa de texto era apresentado um arranjo de estímulos, constituído de um estímulo-modelo (localizado na metade superior da tela) e de três estímulos de comparação (dispostos em fileira, um ao lado do outro, abaixo do estímulo-modelo). Esses estímulos eram figuras geométricas variando em três dimensões: forma (quadrado, círculo, retângulo e triângulo), cor (azul, vermelha e amarela) e espessura (grossa e fina). Estas figuras formavam diferentes arranjos de estímulos. Cada estímulo de comparação apresentava apenas uma dimensão - cor (C), espessura (E) ou forma (F) - em comum com o estímulo-modelo e diferia nas demais. As respostas de escolha emitidas pelos participantes eram registradas automaticamente pelo programa. Os reforçadores eram pontos que eram trocados por dinheiro no final da pesquisa. Cada ponto valia $R \$ 0.10$ (dez centavos de real).

\section{Procedimento}

Primeira Etapa (aplicação do questionário). Na primeira etapa do estudo, após o estudante responder ao questionário, a experimentadora somava as respostas corretas (autorrelatos), de acordo com o gabarito apresentado no texto de Rehfisch (1958). Foram considerados autorrelatos indicativos de flexibilidade aqueles que se encontravam na faixa entre 0 e 11 acertos $(27 \%$ ou menos do total de acertos possíveis) e foram considerados autorrelatos indicativos de inflexibilidade aqueles que se encontravam na faixa entre 29 e 39 acertos ( $75 \%$ ou mais do total de acertos possíveis). Dos 175 participantes, apenas 8 apresentaram autorrelatos dentro da faixa indicativa de inflexibilidade e apenas 8 apresentaram autorrelatos dentro da faixa indicativa de flexibilidade. Todos os participantes concordaram em participar do estudo assinando o Termo de Consentimento Livre e Esclarecido.

Segunda Etapa (exposição às contingências programadas e às regras). No início da sessão experimental, o participante era conduzido até o computador e a expe- rimentadora apresentava oralmente orientações sobre o funcionamento do programa. Em seguida o computador era acionado para dar início à sessão experimental e a experimentadora saía da sala. Em algumas sessões, inicialmente era apresentada ao participante uma determinada instrução e, logo após, eram apresentados os arranjos de estímulos. Em outras sessões, eram apresentados somente os arranjos de estímulos, uma vez que não eram apresentadas instruções.

Em cada tentativa, após ser apresentado um arranjo de estímulos, o participante deveria clicar com o mouse nos três estímulos de comparação em uma dada sequência. Caso a sequência emitida estivesse de acordo com as contingências de reforço programadas (sequência correta), um ponto era então acrescentado no contador (que ficava disponível para o participante na tela do computador), e aparecia a frase "Você ganhou um ponto" na tela. Caso a sequência emitida fosse incorreta, não era acrescentado nenhum ponto no contador, o arranjo desaparecia da tela e um novo arranjo era apresentado. Foi usado um intervalo de 2 segundos entre uma tentativa e outra.

\section{Orientações Preliminares}

Na primeira sessão experimental, eram apresentadas, por meio de uma gravação e também em forma de texto na tela do computador, as seguintes instruções iniciais:

Este objeto que irá piscar aqui em cima é o modelo. Estes três objetos que irão piscar aqui em baixo são para você comparar com o modelo. Vamos chamar estes três objetos de objetos de comparação. Observe que cada um dos objetos de comparação tem apenas uma propriedade em comum com o modelo. Por exemplo, o objeto que irá piscar tem a mesma cor do modelo. E este que irá piscar em seguida tem a mesma forma do modelo. Este que irá piscar agora tem a mesma espessura do modelo. Quando você ganhar pontos, os pontos aparecerão no contador no topo da tela e aparecerá a frase: "Você ganhou um ponto". Quando você não ganhar pontos, nenhum ponto será acrescentado no contador e a frase não será apresentada. Agora vou repetir as instruções para você.

As instruções eram repetidas. Em seguida, o participante ouvia e aparecia na tela o seguinte: "A partir da tela seguinte a pesquisa será iniciada e você poderá começar a tocar".

\section{Delineamento Experimental}

Os 16 participantes foram distribuídos em duas condições experimentais. Quatro participantes que apresentaram autorrelatos classificados de flexíveis e quatro participantes que apresentaram autorrelatos classificados de inflexíveis foram atribuídos à Condição 1 (CRF- ou condição sem variabilidade - replicação sistemática da Condição 2 do Experimento 2 do estudo de Albuquerque et al., 2006). Quatro participantes que apresentaram autorrelatos classificados de flexíveis e quatro participantes que apresentaram autorrelatos classificados de inflexíveis 
foram atribuídos à Condição 2 (FR 4 - ou condição com variabilidade - replicação sistemática do Experimento 2 do estudo de Albuquerque et al., 2003). Cada condição era constituída de quatro fases.

Condição $1(C R F)$. A Fase 1 era iniciada com a apresentação da seguinte instrução mínima:

A sua tarefa será ganhar pontos. Para você ganhar pontos você deve tocar com o dedo em sequência em cada um dos três objetos de comparação. Tente descobrir qual a sequência em que você ganhará pontos. Toda vez que você tocar na sequência correta, você ganhará um ponto no contador que será trocado por $\mathrm{R} \$ 0,10$ (dez centavos de real) ao final da pesquisa. Pode começar a tocar.

Na Fase 1 a sequência de respostas cor, espessura e forma (CEF) era reforçada diferencialmente em CRF até a obtenção de 20 pontos. Depois de estabelecida, esta sequência (CEF) era colocada em extinção em 80 tentativas. Após o critério de 80 tentativas ser atingido, a sequência CEF voltava a ser reforçada até a obtenção de 320 pontos em CRF, quando então esta fase era encerrada.

As Fases 2 e 4 eram iniciadas com a apresentação da seguinte regra discrepante das contingências:

Quando aparecerem estes objetos na tela, você deve fazer o seguinte: Primeiro clique no objeto de comparação que tem a mesma forma do objeto modelo. Depois clique no comparação que tem a mesma cor do objeto modelo. Em seguida clique no objeto que tem a mesma espessura do objeto modelo. Ou seja, você deve tocar primeiro na mesma forma, depois na mesma cor e em seguida na mesma espessura. Entendeu? Clique na tela na sequência descrita. Fazendo isso você poderá ganhar pontos que serão mostrados no contador à sua frente. Cada ponto será trocado por $\mathrm{R} \$ 0,10$ (dez centavos de real) ao final da pesquisa. Pode começar a clicar.

Durante as Fases 2 e 4 o seguimento da regra discrepante (FCE) não era reforçado; somente era reforçada em CRF a emissão da sequência cor, espessura e forma (CEF), a sequência reforçada na Fase 1. A emissão de qualquer outra sequência não era reforçada. Esta fase era encerrada após a obtenção de 80 pontos ou após a ocorrência de 240 tentativas, o que ocorresse primeiro.

A Fase 3 era iniciada com a apresentação da seguinte regra correspondente às contingências:

Quando aparecerem estes objetos na tela, você deve fazer o seguinte: Primeiro clique no objeto de comparação que tem a mesma espessura do objeto modelo. Depois clique no comparação que tem a mesma forma do objeto modelo. Em seguida clique no objeto que tem a mesma cor do objeto modelo. Ou seja, você deve clicar primeiro na mesma espessura, depois na mesma forma e em seguida na mesma cor. Entendeu? Clique na tela na sequência descrita. Fazendo isso você poderá ganhar pontos que serão mostrados no contador à sua frente. Cada ponto será trocado por R\$ 0,10 (dez centavos de real) ao final da pesquisa. Pode começar a clicar.
Durante a Fase 3 a emissão da sequência descrita na regra correspondente, espessura, forma e cor (EFC), bem como a sequência CEF (sequência estabelecida por reforço diferencial na Fase 1), concorrentemente, eram reforçadas em CRF. Esta fase era encerrada após a obtenção de 80 pontos ou após a ocorrência de 240 tentativas, o que ocorresse primeiro.

Condição 2 (FR 4). A Fase 1 da Condição 2 (FR 4) era iniciada com a apresentação de uma instrução mínima idêntica à apresentada na Condição 1 (CRF). Durante a Fase 1 da Condição 2 (FR 4), tal como na Condição 1 (CRF), a sequência reforçada (correta) era cor, espessura e forma (CEF). Inicialmente a sequência correta era reforçada em CRF até serem obtidos 20 pontos em CRF. Em seguida, era feito um aumento gradual no valor do esquema de razão fixa (modelagem) até atingir FR 4 , da seguinte maneira: após a exposição ao esquema CRF, o participante era exposto a um esquema razão fixa 2 (FR 2), até serem obtidos 6 pontos. Imediatamente após, a sequência CEF passava a ser reforçada em razão fixa 3 (FR 3), até serem obtidos 5 pontos. Em seguida, a sequência CEF passava a ser reforçada em razão fixa 4 (FR 4). Neste esquema de razão fixa, a emissão consecutiva da sequência correta produzia um ponto no contador. A não emissão da sequência correta consecutivamente, reiniciava a razão para obtenção de um ponto. O critério de encerramento para esta fase foi a obtenção de 4 pontos consecutivos em FR 4, desde que o participante já tivesse obtido no mínimo 16 pontos em FR 4.

As Fases 2 e 4 da Condição 2 (FR 4) eram iniciadas com a apresentação da regra discrepante das contingências idêntica à apresentada na Condição 1 (CRF). Durante as Fases 2 e 4 da Condição 2 (FR 4), o seguimento de regra discrepante (isto a emissão da sequência FCE) não era reforçado; só era reforçada, em razão fixa 4 (FR4), a emissão da sequência CEF, modelada na Fase 1. Esta fase era encerrada após a obtenção de 20 pontos ou após a ocorrência de 240 tentativas, o que ocorresse primeiro.

A Fase 3 da Condição 2 (FR 4) era iniciada com a apresentação da regra correspondente às contingências idêntica à apresentada na Condição 1 (CRF). Durante esta fase, a emissão da sequência EFC (especificada pela regra correspondente), bem como a emissão da sequência CEF (estabelecida na Fase 1), eram reforçadas em FR 4.

Esta fase era encerrada após a obtenção de 20 pontos ou após a ocorrência de 240 tentativas, o que ocorresse primeiro.

Término da Participação do Estudante no Experimento. A participação do estudante na pesquisa era encerrada após ser atingido o critério de encerramento da última fase da condição a que ele havia sido atribuído, ou caso o participante não atingisse o critério de encerramento da Fase 1.

\section{Resultados}

Na Fase 1, cada um dos 16 participantes atingiu o critério de desempenho para o encerramento dessa fase. 
Paracampo, C. C. P., Souza, L. M. \& Albuquerque, L. C. (2014). Variáveis que Podem Interferir no Seguir Regras de Participantes Flexíveis e Inflexíveis.

Portanto, a sequência cor (C) - espessura - (E) - forma (F) foi estabelecida por reforço diferencial e mantida em CRF na Fase 1 da Condição 1 (CRF) e foi estabelecida por reforço diferencial e mantida em FR 4 na Fase 1 da Condição 2 (FR 4).
A Tabela 1 apresenta os dados da Condição 1 (CRF) e a Tabela 2 apresenta os dados da Condição 2 (FR 4), relativos às porcentagens de sequências de repostas emitidas por cada participante na primeira tentativa e durante toda a fase de cada uma das Fases 2, 3 e 4.

Tabela 1

Porcentagens de Sequências de Respostas na Primeira Tentativa (P) e Durante Toda a Fase (D) de cada uma das Fases 2, 3 e 4 da Condição 1 (CRF)

\begin{tabular}{|c|c|c|c|c|c|c|c|c|c|c|}
\hline & \multirow[b]{3}{*}{ Participantes } & & \multicolumn{8}{|c|}{ Sequências de Respostas } \\
\hline & & & \multicolumn{2}{|l|}{$\mathrm{CEF}$} & \multicolumn{2}{|l|}{ FCE } & \multicolumn{2}{|l|}{$\mathrm{EFC}$} & \multicolumn{2}{|c|}{ Outras } \\
\hline & & & $\mathrm{P}$ & $\mathrm{D}$ & $\mathrm{P}$ & $\mathrm{D}$ & $\mathrm{P}$ & $\mathrm{D}$ & $\mathrm{P}$ & $\mathrm{D}$ \\
\hline \multirow{5}{*}{ Fase 2} & \multirow[t]{4}{*}{ Flexíveis } & P11F & 0 & 94 & 100 & 3 & 0 & 0 & 0 & 3 \\
\hline & & P12F & 0 & 96 & 0 & 3 & 0 & 0 & 100 & 1 \\
\hline & & P13F & 0 & 48 & 100 & 51 & 0 & 0 & 0 & 1 \\
\hline & & P14F & 0 & 95 & 100 & 3 & 0 & 0 & 0 & 2 \\
\hline & \multirow[t]{4}{*}{ Flexíveis } & P11F & 0 & 0 & 0 & 0 & 100 & 100 & 0 & 0 \\
\hline \multirow{3}{*}{ Fase 3} & & P12F & 0 & 0 & 0 & 0 & 100 & 100 & 0 & 0 \\
\hline & & P13F & 0 & 0 & 0 & 0 & 100 & 100 & 0 & 0 \\
\hline & & P14F & 100 & 94 & 0 & 4 & 0 & 1 & 0 & 1 \\
\hline \multirow{5}{*}{ Fase 4} & \multirow[t]{4}{*}{ Flexíveis } & P11F & 0 & 96 & 100 & 3 & 0 & 1 & 0 & 0 \\
\hline & & P12F & 0 & 97 & 100 & 3 & 0 & 0 & 0 & 0 \\
\hline & & P13F & 0 & 90 & 100 & 1 & 0 & 1 & 0 & 8 \\
\hline & & P14F & 100 & 99 & 0 & 1 & 0 & 0 & 0 & 0 \\
\hline & \multirow[t]{4}{*}{ Inflexíveis } & P15I & 0 & 85 & 100 & 5 & 0 & 1 & 0 & 9 \\
\hline \multirow{3}{*}{ Fase 2} & & P16I & 0 & 90 & 100 & 5 & 0 & 0 & 0 & 5 \\
\hline & & P17I & 0 & 92 & 100 & 8 & 0 & 0 & 0 & 0 \\
\hline & & P18I & 100 & 97 & 0 & 3 & 0 & 0 & 0 & 0 \\
\hline \multirow{5}{*}{ Fase 3} & \multirow[t]{4}{*}{ Inflexíveis } & P15I & 0 & 0 & 1 & 0 & 100 & 98 & 0 & 1 \\
\hline & & P16I & 0 & 0 & 0 & 0 & 100 & 100 & 0 & 0 \\
\hline & & P17I & 0 & 0 & 0 & 0 & 100 & 94 & 0 & 6 \\
\hline & & P18I & 0 & 0 & 0 & 0 & 100 & 100 & 0 & 0 \\
\hline & \multirow[t]{4}{*}{ Inflexíveis } & P15I & 0 & 89 & 100 & 7 & 0 & 0 & 0 & 4 \\
\hline \multirow{3}{*}{ Fase 4} & & P16I & 0 & 69 & 0 & 8 & 0 & 7 & 100 & 18 \\
\hline & & P17I & 0 & 97 & 100 & 1 & 0 & 1 & 0 & 1 \\
\hline & & P18I & 0 & 96 & 100 & 2 & 0 & 0 & 0 & 2 \\
\hline
\end{tabular}

Nota. CEF = Sequência estabelecida por reforço diferencial e mantida em esquema de reforço contínuo; FCE = Sequência especificada pela regra discrepente das contingências no início das Fases 2 e 4 . EFC = Sequência especificada pela regra correspondente às contingências no início da Fase 3. 
Passando então a descrever os dados da Condição 1 (CRF), na Tabela 1, observa-se, na Fase 2, quando a regra discrepante das contingências foi apresentada, que 6 (P11F, P13F, P14F, P15I, P16I e P17I) dos 8 participantes da Condição 1 (CRF) iniciaram a Fase 2 seguindo a regra discrepante, isto é, emitindo a sequência FCE na primeira tentativa dessa fase. Mas depois, todos os 8 participantes dessa condição, independentemente de serem classificados de flexíveis ou inflexíveis, passaram a emitir a sequência correta (CEF), que havia sido estabelecida por reforço diferencial na Fase 1. Na Fase 3, quando a regra correspondente às contingências foi apresentada, $7(\mathrm{P} 11 \mathrm{~F}, \mathrm{P} 12 \mathrm{~F}, \mathrm{P} 13 \mathrm{~F}$, P15I, P16I e P17I) dos 8 participantes seguiram esta regra. P14F continuou emitindo CEF. Na Fase 4, quando a regra discrepante voltou a ser apresentada, todos 8 participantes deixaram de seguir a regra discrepante e passaram emitir a sequência correta (CEF) em mais de $69 \%$ das tentativas dessa fase.

Tabela 2

Porcentagens de Sequências de Respostas na Primeira Tentativa (P) e Durante Toda a Fase (D) de cada uma das Fases 2, 3 e 4 da Condição 2 (FR 4)

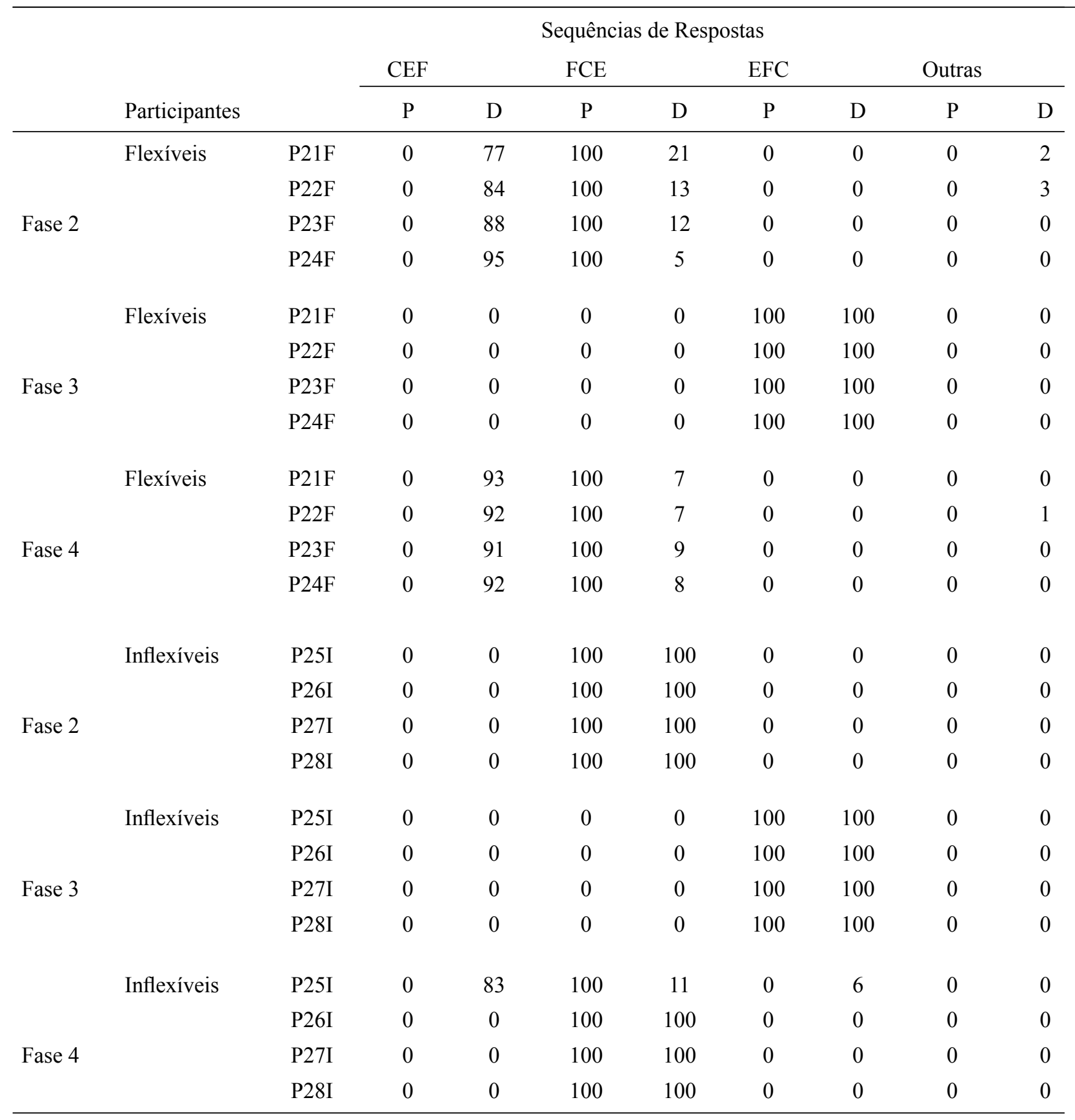

Nota. CEF $=$ Sequência estabelecida por reforço diferencial e mantida em esquema de reforço contínuo; FCE = Sequência especificada pela regra discrepente das contingências no início das Fases 2 e 4. EFC = Sequência especificada pela regra correspondente às contingências no início da Fase 3. 
Paracampo, C. C. P., Souza, L. M. \& Albuquerque, L. C. (2014). Variáveis que Podem Interferir no Seguir Regras de Participantes Flexíveis e Inflexíveis.

Passando agora a descrever os dados da Condição 2 (FR 4), na Tabela 2, pode-se observar que, na Fase 2, os 4 participantes inflexíveis (P25I, P26I, P27I e P28I) seguiram a regra discrepante das contingências, enquanto os 4 participantes flexíveis (P21F, P22F, P23F e P24F) deixaram de seguir esta regra. Na Fase 3, todos os 8 participantes, independentemente de serem classificados de flexíveis ou inflexíveis, seguiram a regra correspondente. Na Fase 4, os 4 participantes flexíveis deixaram de seguir a regra discrepante e 3 (P26I, P27I e P28I) dos 4 participantes inflexíveis seguiram a regra discrepante. Portanto, 5 (P21F, P22F, P23F, P24F e P25I) dos 8 participantes deixaram de seguir a regra discrepante na Fase 4.

\section{Discussão}

O presente estudo procurou verificar se os efeitos de histórias pré-experimentais, inferidas das respostas dos participantes ao questionário sobre inflexibilidade, dependem da situação atual, à qual uma pessoa é exposta.

Os resultados da Condição 1 (CRF) do presente estudo mostraram que a situação atual, à qual uma pessoa é exposta, pode impedir os eventuais efeitos de histórias pré-experimentais na determinação de diferenças individuais. Os resultados dessa condição replicam os resultados da Condição 2 do Experimento 2 do estudo de Albuquerque e colaboradores (2006), visto que todos os participantes dessas duas condições, sem exceção, deixaram de seguir a regra discrepante das contingências nas Fases 2 e 4 . Mas em adição, os resultados da Condição 1 (CRF) do presente estudo, de modo similar aos resultados encontrados por Pinto e colaboradores (2008), mostraram que todos os participantes deixaram de seguir a regra discrepante, independentemente das diferenças entre as suas histórias pré-experimentais, isto é, independentemente de se os participantes eram flexíveis ou inflexíveis.

Isto não implica que as histórias pré-experimentais dos participantes não possam interferir em seus desempenhos. Os resultados da Condição 2 (FR 4) replicaram, em grande parte, os resultados do Experimento 2 do estudo de Albuquerque e colaboradores (2003). Neste estudo de 2003, $67 \%$ (4 de 6) dos participantes seguiram a regra discrepante nas Fases 2 e 4, enquanto na Condição 2 (FR 4) do atual estudo, $63 \%$ (5 de 8 ) e $37 \%$ ( 3 de 8 ) dos participantes seguiram a regra discrepante das contingências nas Fases 2 e 4, respectivamente. A diferença foi que, na Condição 2 (FR 4) do atual estudo, todos os participantes que seguiram a regra discrepante eram inflexíveis e, com exceção de um participante inflexível (P25I) que deixou de seguir a regra discrepante na Fase 4, todos os demais participantes que não seguiram esta regra eram flexíveis. Estes dados são similares aos encontrados por Pinto e colaboradores (2006) e Wulfert e colaboradores (1994) os quais indicam que as diferenças entre os desempenhos dos participantes, dentro de uma mesma condição experimental, podem ser atribuídas, em parte, às diferenças entre as suas histórias pré-experimentais, uma vez que os participantes previamente classificados de flexíveis tenderam a não seguir a regra discrepante e os participantes previamente classificados de inflexíveis tenderam a seguir a regra discrepante.

Os dados da Condição 2 (FR 4) apoiam, portanto, a sugestão de que as histórias pré-experimentais podem interferir na ocorrência de diferenças individuais em determinadas situações (Weiner, 1983). Contudo, isto não implica que as diferenças entre os desempenhos encontradas na Condição 2 (FR 4) devam ser atribuídas, exclusivamente, às diferenças entre as histórias pré-experimentais dos participantes. Se as diferenças entre os desempenhos dos participantes, observadas na Fase 2 da Condição 2 (FR 4), tivessem ocorrido devido às diferenças entre as histórias pré-experimentais dos participantes (isto é, devido ao fato de uns serem flexíveis e outros serem inflexíveis), então, tais diferenças também deveriam ter ocorrido na Fase 3 das duas condições e na Fase 2 da Condiçãol (CRF); e isto não ocorreu.

Similarmente, também não se pode dizer que os Participantes P25I, P26I, P27I e P28I seguiram a regra discrepante, porque o esquema que reforçava o seguimento e o não seguimento de regra era um esquema de reforço intermitente (Newman et al., 1995). Se o esquema fosse o único fator a determinar o desempenho dos participantes, P21F, P22F, $\mathrm{P} 23 \mathrm{~F}$ e $\mathrm{P} 24 \mathrm{~F}$ teriam seguido a regra discrepante; o que não ocorreu. Do mesmo modo, também não se pode dizer que os participantes P21F, P22F, P23F e P24F deixaram de seguir a regra discrepante, porque as contingências programadas a que foram expostos eram fortes, isto é, porque foi demonstrado controle por contingências antes da apresentação da regra (Torgrud \& Holborn, 1990). Se apenas este fator fosse suficiente para determinar os desempenhos dos participantes, P25I, P26I, P27I e P28I teriam deixado de seguir a regra discrepante; o que não ocorreu.

Contudo, pode-se sugerir que a história pré-experimental contribuiu para que 14 (P11F, P13F, P14F, P15F, P16F, P17F, P21F, P22F, P23F, P24F, P25I, P26I, P27I e P28I) dos 16 participantes do presente estudo tivessem deixado de emitir o comportamento que vinha produzindo reforço em suas histórias experimentais e tivessem emitido o comportamento especificado pela regra discrepante, na transição da Fase 1 para a Fase 2. Mas a situação, à qual os participantes foram expostos, também contribuiu para determinar os seus desempenhos.

Neste ponto, dois aspectos devem ser considerados. O primeiro é que, a partir da Fase 2, inclusive, a topografia do comportamento apresentado pelos participantes foi determinada, ou pelas regras (como ocorreu, por exemplo, nos casos de P26I, P27I e P28I, que passaram a emitir as sequências FCE, EFC e FCE, nas Fases 2, 3 e 4, respectivamente, em função das mudanças nas regras), ou pela história experimental de reforço do comportamento alternativo ao especificado pela regra (como ocorreu, por exemplo, nos casos em que os participantes deixavam de seguir uma regra e passavam a emitir a sequência [CEF] estabelecida na Fase 1). E segundo, o comportamento foi mantido sob controle de regra ou sob controle da história de reforço do comportamento alternativo, não devido a uma ou outra variável particular, mas sim devido à combinação 
entre as situações que favoreciam e as que não favoreciam o seguimento e o não seguimento de regras (Albuquerque et al., 2003; Oliveira \& Albuquerque, 2007).

No presente estudo, a situação que mais favorecia o seguimento de regra era a Fase 3 das duas condições, porque nesta situação o seguimento da regra correspondente às contingências produzia as consequências descritas na regra ( 15 dos 16 participantes, independentemente da condição experimental e de se o participante era flexível ou inflexível, seguiram a regra correspondente). Esta situação, portanto, pouco favorecia os efeitos de histórias pré-experimentais na determinação de diferenças individuais.

Por outro lado, a situação que mais favorecia o não seguimento de regra (isto é, a ocorrência do comportamento sob o controle da história de reforço do comportamento alternativo) era a Fase 2 da Condição 1 (CRF), porque nesta situação: (a) o seguimento da regra discrepante produzia consequências que contradiziam a regra (a não obtenção de pontos); (b) o não seguimento de regra era reforçado em CRF, ou seja, o participante tinha que emitir apenas uma determinada sequência de respostas para que esta sequência fosse reforçada em CRF; e, (c) os participantes tinham uma história em que o comportamento alternativo ao especificado pela regra havia sido estabelecido por reforço diferencial e mantido em CRF (Albuquerque et al., 2006; Albuquerque et al., 2003). Esta situação também não favorecia os efeitos de histórias pré-experimentais na determinação de diferenças individuais.

A Fase 2 da Condição 2 (FR 4) era a situação mais equilibrada quanto às variáveis que favoreciam o seguimento e o não seguimento de regra. Essa situação favorecia o não seguimento de regra porque o seguimento da regra discrepante produzia consequências que contradiziam a regra. Mas, por outro lado, também favorecia o seguimento de regra, porque o comportamento de não seguir regra envolvia alto custo de resposta, uma vez que persistir no comportamento de não seguir a regra implicava em claramente desobedecer ao experimentador. Ou seja, para o não seguimento de regra poder ser reforçado, o participante tinha que responder quatro vezes consecutivas na sequência correta e a não emissão consecutiva da mesma sequência correta, reiniciavam a razão fixa para a obtenção de um ponto. Além disso, os participantes tinham uma história em que o comportamento tinha que persistir, na ausência de reforço, para só então ele poder ser reforçado. Essa história pode ter estabelecido, não apenas a sequência correta, mas também o comportamento de persistir. Essa característica da história da Fase 1 desses participantes pode ter interferido na persistência dos comportamentos observados na Fase 2. Assim, essa história pode ter contribuído para que alguns participantes (caso de P21F, P22F, P23F e P24F) tivessem persistido no comportamento de não seguir a regra e para que outros (caso de P25I, P26I, P27I e P28I) tivessem persistido no comportamento de seguir a regra (Albuquerque et al., 2003; Oliveira \& Albuquerque, 2007). Portanto, esta situação era a que mais favorecia os efeitos de histórias pré-experimentais na determinação de diferenças individuais.
Esta análise sugere, então, que os efeitos de histórias pré-experimentais na determinação de diferenças individuais dependem, em grande parte, da situação atual à qual as pessoas são expostas. Ou seja, é a situação atual que favorece, ou não, os efeitos de histórias pré-experimentais na determinação de diferenças individuais.

Considerando esta análise, pode-se dizer, então, que os resultados do presente estudo (mais especificamente os resultados da Fase 2 da Condição 2 [FR 4]) apoiam a sugestão dos estudos anteriores (Pinto et al., 2006, 2008; Wulfert et al.,1994), de que se pode inferir, a partir das respostas dos participantes aos itens do questionário desenvolvido por Rehfisch (1958), que participantes flexíveis e inflexíveis tem diferentes histórias pré-experimentais de seguir regras. Mas, como Pinto e colaboradores (2008) destacaram, tais respostas ao questionário não indicam o tipo específico de história de seguir regras de cada participante, isto é, apenas com base em tais dados, não se pode identificar se a história pré-experimental do participante era de reforço social para o seguir regras similares (Skinner, 1974), ou de reforço social em algumas situações que se generalizou para outras situações (Catania, 1998), ou de punição social do não seguir regras (Wulfert et al., 1994), ou uma história de exposição a regras que relatam promessas de reforço para o seguir regras e ameaças de punição para o não seguir regras (Albuquerque, 2005; Albuquerque \& Paracampo, 2010). Contudo, independentemente do tipo de história pré-experimental de seguir regras de cada participante, os resultados do presente estudo sugerem que os eventuais efeitos de tal história dependem de variáveis atuais. Uma implicação disso é que o controle por regras não pode ser explicado apenas com base em uma história pré-experimental de seguir regras. Em outras palavras, as proposições sobre por que regras são seguidas que enfatizam apenas os efeitos de histórias pré-experimentais não são suficientes para explicar o controle por regras. Portanto, para explicar o controle por regras, também é necessário considerar os efeitos das variáveis atuais, tal como foi feito no presente estudo.

Finalmente, os resultados do presente estudo apoiam a proposição de Albuquerque e Paracampo (2010) acerca da distinção entre o controle por regras e o controle por contingências. De acordo com estes autores, pode-se dizer que o comportamento é controlado por regra quando a sua topografia é estabelecida por uma regra e ele ocorre independentemente de suas consequências imediatas, tal como ocorreu na Fase 2 de P25I, por exemplo. Pode-se dizer que um comportamento é controlado por contingências quando a sua topografia é estabelecida por suas consequências imediatas e ele ocorre independentemente de regras (tal como ocorreu na Fase 2 [após o seguimento de regra ter deixado de ocorrer] de P11F e P15I, por exemplo). O comportamento estabelecido por regra pode depender de suas consequências imediatas, mas quando isto ocorre, este comportamento deixa de ser puramente controlado por regra e passa a ser controlado, ou por contingências (tal como ocorreu na Fase 2 [após o seguimento de regra ter deixado de ocorrer] de P11F e P15I, por exemplo) ou 
Paracampo, C. C. P., Souza, L. M. \& Albuquerque, L. C. (2014). Variáveis que Podem Interferir no Seguir Regras de Participantes Flexíveis e Inflexíveis.

controlado pela interação entre regra e contingência (tal como ocorreu na Fase 3 de P11F e P15I, por exemplo).

Os dados do presente estudo também mostram como regras e contingências funcionam. Utilizando os dados do Participante P26I como exemplo, pode-se dizer que na Fase 1, a topografia do comportamento (isto é, a emissão da sequência cor-espessura-forma por quatro vezes consecutivas) foi estabelecida por suas consequências imediatas, enquanto que na Fase 2 a topografia do comportamento (isto é, a emissão da sequência forma-cor-espessura por quatro vezes consecutivas) foi estabelecida pela regra. $\mathrm{Na}$ Fase 1 as consequências imediatas programadas no experimento estabeleceram a função discriminativa das dimensões do estímulo modelo e dos estímulos de comparação. Na Fase 2, a função discriminativa de tais dimensões foi estabelecida pela regra. Uma implicação destes dados é que regras não deveriam ser classificadas como estímulos discriminativos, nem como operações estabelecedoras, porque estímulos discriminativos e operações estabelecedoras não determinam a topografia do comportamento e regras podem exercer esta função. Neste sentido, regras teriam uma função similar à exercida pelas consequências do comportamento. Mas diferente das consequências, regras podem evocar comportamento. Deste modo, os efeitos de regras são similares aos de contingências. Mas diferente de contingências, regras podem estabelecer um comportamento novo, independentemente de suas consequências imediatas (Albuquerque \& Paracampo, 2010)

Esta análise sugere que o comportamento especificado pela regra pode variar em função de variações nas propriedades formais da regra (isto é, de variações nos estímulos que constituem a regra). Como tais estímulos também fazem parte das variáveis atuais que interferem no seguir regras, pesquisas futuras deveriam avaliar, por exemplo, se as consequências relatadas na regra (no presente estudo, a consequência relatada era a promessa de que o participante ganharia pontos trocáveis por dinheiro), podem alterar a probabilidade de o comportamento especificado pela regra vir a ocorrer no futuro. Pesquisas futuras poderiam avaliar, por exemplo, os efeitos de manipulações de diferentes tipos consequências relatadas na regra sobre o seguir regras.

\section{Referências}

Albuquerque, L. C. (2001). Definições de regras. In H. J. Guilhardi, M. B. B. P. Madi, P. P. Queiroz, \& M. C. Scoz (Eds.), Sobre comportamento e cognição: Expondo a variabilidade (pp.132-140). Santo André, SP: ARBytes.

Albuquerque, L. C. (2005). Regras como instrumento de análise do comportamento. In L. C. Albuquerque (Ed.), Estudos do comportamento (pp.143-176). Belém, PA: Editora da Universidade Federal do Pará.

Albuquerque, L. C., \& Paracampo, C. C. P. (2010). Análise do controle por regras. Psicologia USP, 21, 253-273.

Albuquerque, L. C., \& Silva, F. M. (2006). Efeitos da exposição a mudanças nas contingências sobre o seguir regras. Psicologia: Teoria e Pesquisa, 22, 101-112.

Albuquerque, L. C., Reis, A. A., \& Paracampo, C. C. P. (2006). Efeitos de uma história de reforço contínuo sobre o seguimento de regra. Acta Comportamentalia, 14, 47-75.
Albuquerque, L. C., de Souza, D. G., Matos, M. A., \& Paracampo, C. C. P. (2003). Análise dos efeitos de histórias experimentais sobre o seguimento subsequente de regras. Acta Comportamentalia, 11, 87-126.

Baron, A., \& Galizio, M. (1983). Instructional control of human operant behavior. The Psychological Record, 33, 495-520.

Catania, A. C. (1998). Learning (4 ${ }^{\text {th }}$ ed.). Upper Saddle River, NJ: Prentice-Hall.

Hayes, S. C., Brownstein, A. J., Zettle, R. D., Rosenfarb, I., \& Korn, Z. (1986). Rule governed behavior and sensitivity to changing consequences of responding. Journal of the Experimental Analysis of Behavior, 45, 237-256.

LeFrancois, J. R., Chase, P. N., \& Joyce, J. (1988). The effects of variety of instructions on human fixed-interval performance. Journal of the Experimental Analysis of Behavior, 49, 383-393.

Newman, B., Buffington, D. M., \& Hemmes, N. S. (1995). The effects of schedules of reinforcement on instruction following. The Psychological Record, 45, 463-476.

Oliveira, V. L., \& Albuquerque, L. C. (2007). Efeitos de histórias experimentais e de esquemas de reforço sobre o seguir regras. Psicologia: Teoria e Pesquisa, 23, 217-228.

Paracampo, C. C. P., \& Albuquerque, L. C. (2004). Análise do papel das consequências programadas no seguimento de regras. Interação em Psicologia, 8, 237-245.

Paracampo, C. C. P., \& Albuquerque, L. C. (2005). Comportamento controlado por regras: Revisão crítica de proposições conceituais e resultados experimentais. Interação em Psicologia, 9, 227-237.

Pinto, A. R., Paracampo, C. C. P., \& Albuquerque, L. C. (2006). Análise do controle por regras em participantes classificados de flexíveis e de inflexíveis. Acta Comportamentalia, 14, 171-194.

Pinto, A. R., Paracampo, C. C. P., \& Albuquerque, L. C. (2008). Efeitos de perda de reforçadores sobre o seguir regras em participantes flexíveis e inflexíveis. Revista Brasileira de Análise do Comportamento, 1, 111-125.

Rehfisch, J. M. (1958). A Scale for Personality rigidity. Journal of Consulting Psychology, 1, 11-15.

Shimoff, E., Catania, A. C., \& Matthews, B. A. (1981). Uninstructed human responding: Sensitivity of low-rate performance to schedule contingencies. Journal of the Experimental Analysis of Behavior, 36, 207-220.

Skinner, B. F. (1974). About behaviorism. New York: Alfred A. Knopf.

Torgrud, L. J., \& Holborn, S. W. (1990). The effects of verbal performance descriptions on nonverbal operant responding. Journal of the Experimental Analysis of Behavior, 54, 273-291.

Weiner, H. (1983). Some thoughts on discrepant human-animal performances under schedules of reinforcement. The Psychological Record, 33, 521-532.

Wulfert, E., Greenway, D. E., Farkas, P., Hayes, E. C., \& Douguer, M. J. (1994). Correlation between self-reported rigidity and rule-governed insensitivity to operant contingencies. Journal of Applied Behavior Analysis, 27, 659-671. 